\title{
SHAPE-CONSTRAINED SEGMENTATION APPROACH FOR ARCTIC MULTIYEAR SEA ICE FLOE ANALYSIS
}

\author{
Yuliya Tarabalka ${ }^{1,2}$, Ludovic Brucker ${ }^{2,3}$, Alvaro Ivanoff ${ }^{2}$, James C. Tilton ${ }^{2}$ \\ ${ }^{1}$ INRIA, AYIN team, 2004 route des Lucioles, 06902 Sophia Antipolis, France. \\ ${ }^{2}$ NASA Goddard Space Flight Center, MD, USA. ${ }^{3}$ Universities Space Research Association, MD, USA. \\ e-mail: yuliya.tarabalka@inria.fr
}

\begin{abstract}
The melting of sea ice is correlated to increases in sea surface temperature and associated climatic changes. Therefore, it is important to investigate how rapidly sea ice floes melt. For this purpose, a new TempoSeg method for multitemporal segmentation of multiyear ice floes is proposed. The microwave radiometer is used to track the position of an ice floe. Then, a time series of MODIS images are created with the ice floe in the image center. A TempoSeg method is performed to segment these images into two regions: Floe and Background. First, morphological feature extraction is applied. Then, the central image pixel is marked as Floe, and shape-constrained best merge region growing is performed. The resulting tworegion map is post-filtered by applying morphological operators. We have successfully tested our method on a set of MODIS images and estimated the area of a sea ice floe as a function of time.
\end{abstract}

Index Terms - Classification, segmentation, region merging, multiyear sea ice floes, shape analysis.

\section{INTRODUCTION}

The sea ice cover has a significant effect on the Earth's climate system. It limits the exchange of heat, moisture, and momentum across the ocean-sea ice-snow-atmosphere interface. Therefore, sea ice is both an active participant of the Earth's climate and a sensitive climate indicator [1]. It thus is very important to monitor sea ice evolution and develop methods for automated analysis of satellite measurements. In this paper, our objective is to determine how rapidly a multiyear ice floe can melt.

Most of the existing techniques for automatic ice floe tracking from Synthetic Aperture Radar or optical remote sensing imagery employ adaptive, or dynamic thresholding for distinguishing ice from water [2, 3]. In [4], Yu applied iterative region growing to the segmentation of sea ice imagery from the RADARSAT-2 data. A watershed transform was performed, followed by labeling of the resulting homogeneous regions and region merging to obtain an accurate segmentation map.
In our study, two sensors onboard the NASA Aqua satellite have been used: Advanced Microwave Scanning Radiometer - Earth Observing System (AMSR-E) and ModerateResolution Imaging Spectroradiometer (MODIS). AMSR-E data was used to manually track the position of the ice floe of interest. Based on this information, a time series of the reprojected MODIS images was created with the ice floe in the central part of every image. These measurements made it possible to estimate both an area and a perimeter of a multiyear Arctic sea ice floe as a function of time using a new multitemporal segmentation (TempoSeg) method. The proposed TempoSeg method is based on shape-constrained best merge region growing and proceeds as follows:

First, a feature extraction by applying morphological operators is applied. Every pixel is considered as a separate region. The central image region is assigned to the class Floe, while all other regions are assigned to the class Background. Second, hierarchical step-wise optimization segmentation [5] is performed, by iteratively merging two adjacent regions with the smallest Dissimilarity Criterion (DC). When any Background region is merged with the Floe region, the resulting region is assigned to the class Floe. The algorithm is converged for a given image when the image is segmented into two regions: Floe and Background. Finally, a morphological post-filtering of the resulting map is applied.

The important contribution of this work consists in including multitemporal, geometrical and shape analysis for computing a DC between two regions. Moreover, this method is applied on several images processed sequentially.

The outline of the paper is as follows. In the next section, the data set used for experiments is described. Section 3 presents the proposed TempoSeg segmentation method. Experimental results are discussed in Section 4. Finally, conclusions are drawn in Section 5.

\section{DATA SET}

In this study, we analyzed two months of AMSR-E and MODIS measurements. These sensors are onboard the Aqua satellite, which has a polar orbit enabling several measure- 


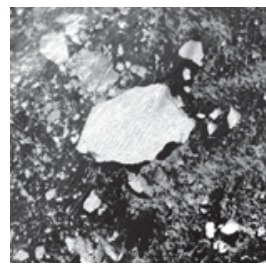

(a)

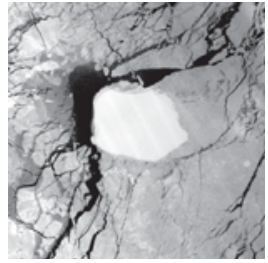

(c)

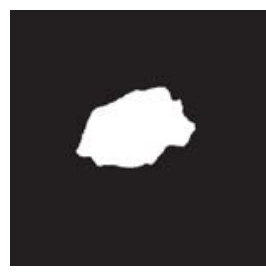

(b)

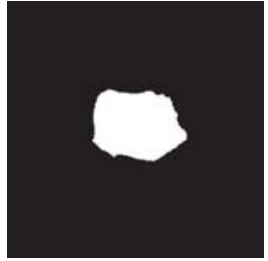

(d)

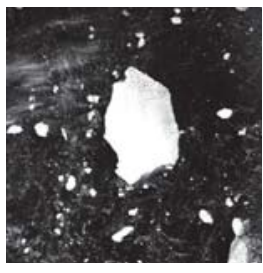

(a)

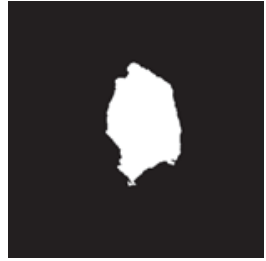

(c)

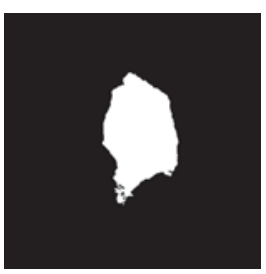

(b)

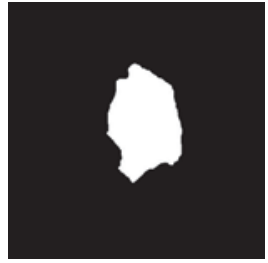

(d)
Fig. 1. (a) Reprojected MODIS I(227-1435) image. (b) TempoSeg segmentation map of I(227-1435) (floe area $=24104$ pixels). (c) Reprojected MODIS I(286-2045) image. (d) Segmentation map of $I(286-2045)$ (floe area $=19315$ pixels $)$.

ments per day over the Earth's polar regions. Our target of interest is a multiyear sea ice floe, which left the Arctic main sea ice pack in December 2007, continuously moved according to the Arctic ocean currents and melted in June 2009.

To track the position of the ice floe of interest during its lifetime, the AMSR-E data at $89 \mathrm{GHz}$ mapped to a polar stereographic grid at $6.25 \mathrm{~km}$ spatial resolution were used. These data have been extracted from the "AMSR-E/Aqua Daily L3 6.25 km 89 GHz Brightness Temperature Polar Grids" product distributed by the National Snow and Ice Data Center. In accordance with these measurements, a time series of the MODIS images was built with the ice floe of interest.

In order to get the most accurate segmentation results, it is the best to use the highest MODIS spatial resolution band at $250 \mathrm{~m}$. Among the two high-resolution bands of the MODIS data, we used band $1(0.620-0.670 \mu \mathrm{m})$ because it provided the best contrast between the sea ice and the ocean water. Level 1B calibrated and geolocated MODIS swath data reprojected onto a polar stereographic grid was the main data source. The geolocation uncertainty is $\sim 50 \mathrm{~m} \mathrm{[6]}$.

All swath images did not provide opportunities to quantify the ice floe areas either due to extensive cloud cover, limited solar illumination, or weakness of contrast between the multiyear ice floe and the surrounding young ice. We selected for our analysis $M=40$ images with spatial dimensions of 500 $\times 500$ pixels, acquired on 18 different days during the twomonths period from mid-August to mid-October 2008 (from the 227th day to the 286th day of 2008). Fig. 1(a,c) and 2(a) show three images from the considered set. We denote each image by $I_{m}(D-T)=\left\{i_{m}(x, y) \in \mathbb{Z}^{+}, x=[1 . . H], y=\right.$ $[1 . . W]\}, m=[1 . . M]$, where $D$ and $T$ mean the day and the
Fig. 2. Image I(249-1715): (a) Reprojected MODIS image. (b) Segmentation map when no shape constraints and no post-filtering were applied (floe area $=23822$ pixels). (c) TempoSeg segmentation map before post-filtering (floe area = 22903 pixels). (d) TempoSeg final segmentation map (floe area $=22853$ pixels $)$.

time when it was recorded, $H$ and $W$ are height and width of each image, respectively (in our case, $H=W=500$ ). For instance, the image $I_{1}(227-1435)$ was acquired on the day 227 at $14: 35$.

\section{PROPOSED METHOD}

The proposed TempoSeg segmentation method is based on shape-constrained best merge region growing procedure. It consists of the following steps:

Input: At the input, $M$ 1-band images $I_{m}(D-T)=$ $\left\{i_{m}(x, y) \in \mathbb{Z}^{+}, x=[1 . . H], y=[1 . . W]\right\}, m=[1 . . M]$ including the Floe are given. The goal is to obtain for each image $I_{m}$ a segmentation map $S_{m}=\left\{s_{m}(x, y) \in[0,1], x=\right.$ $[1 . . H], y=[1 . . W]\}$, where each pixel has label 1 if it belongs to the Floe, and 0 otherwise. The following parameters must be initialized: maximum number of pixels representing the floe $N \max _{F}$, maximum height $H \max _{F}$, maximum width $W \max _{F}$ of the flow, respectively, and its rectangularity at the previous time moment $\operatorname{Rect}_{F}^{0}$.

We define a height $H \operatorname{eight}(R)$ and a width $W i d t h(R)$ of the region $R$ as the height and the width of the Minimum Area Rectangle (MAR) of an arbitrary orientation including $R$. A region rectangularity $\operatorname{Rect}(R)$ is computed as a ratio of the region area (i.e. number of pixels) and the area of the MAR enclosing $R$ (i.e. Height $(R) \cdot W i d t h(R)$ ).

Feature extraction: In order to enhance the difference between the intensity values of the Floe and the rest of the image, each image $I_{m}$ is filtered to remove small objects, as described in the following: 
1. Morphological $h$-minima transform is applied to $I_{m}$, which suppresses all minima in the image whose depth is less than a scalar $h$ [7], resulting in an image $I_{m}^{1}$.

2. $H$-maxima transform is applied to $I_{m}^{1}$, which suppresses all maxima whose height is less than $h$, resulting in an image $I_{m}^{2}$ [7].

3. Geodesic opening by reconstruction $I_{m}^{3}=\gamma\left(I_{m}^{2}\right)$ of the current image is obtained by reconstructing the image erosion $\varepsilon\left(I_{m}^{2}\right)$ under $I_{m}^{2}$, using a disk with radius $r$ as the structuring element (SE) [7]. This operation removes bright objects with a thinner support than the given SE, while preserving shapes of other objects.

4. Geodesic closing by reconstruction $I_{m}^{4}=\varphi\left(I_{m}^{3}\right)$ is performed in the similar way, with a disk of radius $r$ as a SE [7].

The obtained image $I_{m}^{4}$ is combined with the original image $I_{m}$, resulting in a 2-band image $\mathbf{I}_{m}=\left\{\mathbf{i}_{m}(x, y) \in\right.$ $\left.\mathbb{Z}^{2}, x=[1 . . H], y=[1 . . W]\right\}$.

Hierarchical optimization: The segmentation by hierarchical shape-constrained optimization of each image $\mathbf{I}_{m}$ is sequentially performed as follows:

1. $m=1$.

2. Consider the image $\mathbf{I}_{m}$. Initialize the algorithm by assigning a new region label for each pixel. The one-pixel region situated in the center of the image [with coordinates $(\mathrm{H} / 2+1, \mathrm{~W} / 2+1)]$ gets a Floe class label $L\left(R_{F}\right)=1$. All other regions get a class label $L\left(R_{j}\right)=0, j=[1 . . W \times$ $H], j \neq F$.

3. Calculate the DC between all pairs of spatially adjacent regions $\left\{R_{j}, R_{k}\right\}$, considering an eight-connectivity neighborhood and using the following algorithm (see Fig. 3):

- Compute the square root of band sum mean squared error $\left(\mathrm{BSMSE}^{1 / 2}\right)$ criterion, which is based on minimizing the increase of mean squared error between the region mean image and the original image data. The BSMSE $^{1 / 2}$ between regions $R_{j}$ and $R_{k}$ with region mean vectors $\mathbf{u}_{j}=\left(u_{j 1}, u_{j 2}\right)^{T}$ and $\mathbf{u}_{k}=\left(u_{k 1}, u_{k 2}\right)^{T}$ and region sizes (number of pixels) $n_{j}$ and $n_{k}$, respectively, is defined as

$$
\begin{gathered}
D C\left(R_{j}, R_{k}\right)=B S M S E^{\frac{1}{2}}\left(R_{j}, R_{k}\right)= \\
{\left[\frac{n_{j} n_{k}}{\left(n_{j}+n_{k}\right)} \sum_{b=1}^{2}\left(u_{j b}-u_{k b}\right)^{2}\right]^{1 / 2} .}
\end{gathered}
$$

- If either $L\left(R_{j}\right)=1$ or $L\left(R_{k}\right)=1$, i.e., one of the regions represents a floe, proceed as follows:

a) If $\left(n_{j}+n_{k}\right)>N \max _{F}, D C\left(R_{j}, R_{k}\right)=\infty$ (the upper maximum value of float).

b) Otherwise, if $\left(n_{j}+n_{k}\right)>K \cdot N \max _{F}$, $0<K<1$, perform shape analysis. The parameter $K$ controls the scale at which a shape analysis

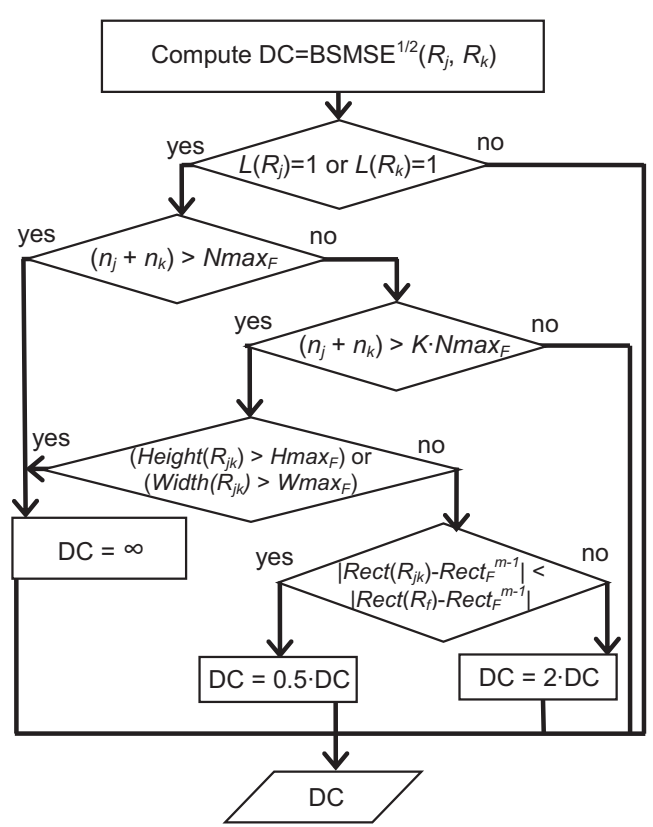

Fig. 3. Flowchart of computing a DC between two regions $R_{j}$ and $R_{k} . R_{j k}=R_{j} \cup R_{k}$, and $f \in\{j, k\}, L\left(R_{f}\right)=1$.

is started.

Let $R_{j k}=R_{j} \cup R_{k}$, and $f \in\{j, k\}, L\left(R_{f}\right)=1$.

- If $\left(\operatorname{Height}\left(R_{j k}\right)>\operatorname{Hmax}_{F}\right)$ or $\left(W i d t h\left(R_{j k}\right)>W \max _{F}\right)$, then $D C\left(R_{j}, R_{k}\right)=\infty$.

- Otherwise, if

$$
\begin{aligned}
\left|\operatorname{Rect}\left(R_{j k}\right)-\operatorname{Rect}_{F}^{m-1}\right| & <\left|\operatorname{Rect}\left(R_{f}\right)-\operatorname{Rect}_{F}^{m-1}\right|, \\
\text { then } D C\left(R_{j}, R_{k}\right) & =0.5 D C\left(R_{j}, R_{k}\right), \\
\text { else } D C\left(R_{j}, R_{k}\right) & =2 D C\left(R_{j}, R_{k}\right) .
\end{aligned}
$$

The idea behind this rectangularity condition is based on an assumption that the time interval between acquisitions of two consecutive images is typically short enough, so that the rectangularity values of the Floe region within these two images are similar.

4. Find the smallest $\mathrm{DC}$ value $D C_{\text {min }}$.

5. Merge a pair of spatially adjacent regions with $D C=$ $D C_{\min }$. If one of the merged regions has a class label 1 , a new region gets a class label 1 , and its rectangularity value $\operatorname{Rect}\left(R_{f}\right)$ is recomputed.

6. If the image is segmented into two regions Floe and Background, go to step 7. Otherwise, update the DC values between the new region and all regions spatially adjacent to it, and return to step 4.

7. Update parameters:

- $\operatorname{Rect}_{F}^{m}=0.5\left(\operatorname{Rect}_{F}^{m-1}+\operatorname{Rect}_{F}^{m}\right)$.

- If $m>1$ : 


$$
\begin{aligned}
& N \max _{F}=\min \left[N \max _{F}, 1.05 \max \left(n_{F}^{m-1}, n_{F}^{m}\right)\right] . \\
& \operatorname{Hmax}_{F}=\min \left[\operatorname{Hmax}_{F}\right. \text {, } \\
& \left.1.2 \max \left(\operatorname{Height}\left(R_{F}^{m-1}\right), \operatorname{Height}\left(R_{F}^{m}\right)\right)\right] . \\
& W \max _{F}=\min \left[W \max _{F},\right. \\
& \left.1.2 \max \left(\operatorname{Width}\left(R_{F}^{m-1}\right), \operatorname{Width}\left(R_{F}^{m}\right)\right)\right] . \\
& \text { 8. } m=m+1 \text {. Go to step } 2 \text {. }
\end{aligned}
$$

Post-filtering: Each segmentation map $S_{m}$ is post-filtered by applying the following morphological operators [7]:

- Opening with a disk of radius $r_{o}$ as a SE.

- Area opening to remove all small Floe regions that may appear after morphological opening.

- Closing using a disk with radius $\left(r_{o}+2\right)$ as a SE.

\section{EXPERIMENTAL RESULTS AND DISCUSSION}

We applied the proposed TempoSeg approach to the set of 40 MODIS images described in Section 2. Based on the visual analysis of the first image in a time series $I_{1}(227-1435)$ (see Fig. 1(a)), we initialized the parameters $N \max _{F}=27000$, $\operatorname{Hmax}_{F}=250, W \max _{F}=170$ and Rect ${ }_{F}^{0}=0.71$ (compare to the floe characteristics we estimated by applying TempoSeg: $n_{F}^{1}=24104, \operatorname{Height}\left(R_{F}^{1}\right)=232, \operatorname{Width}\left(R_{F}^{1}\right)=$ $\left.145, \operatorname{Rect}_{F}^{1}=0.72\right)$. Other parameters were empirically set as $h=30, r=15, K=0.8$ and $r_{o}=4$.

Fig. 1(b, d) and 2(b-d) show some of the obtained segmentation images. From these images, it is possible to see that the proposed approach succeeded in accurately segmenting the ice floe of interest from the rest of the image. Fig. 2 demonstrates the improvement in segmentation results when including shape analysis into best merge region growing (compare Fig. 2(c) versus Fig. 2(b)) and when applying morphological post-filtering (compare Fig. 2(d) versus Fig. 2(c)).

Fig. 4 depicts graphs of the Floe area and perimeter as a function of time. As expected, during the two-month period at the end of the summer season, both area and perimeter are decreasing over time. Some variability such as the local minima and maxima of the curves are a consequence of either a partial cloud cover over the Floe (minima) in some images, or weakness of contrast between the multiyear ice and the neighboring young ice floes (maxima).

\section{CONCLUSIONS}

We have designed a new TempoSeg method for multitemporal segmentation of multiyear sea ice floes from the MODIS data. The proposed technique is based on shape-constrained best merge region growing, and it segments each image from a time series into Floe and Background regions. We have applied this method to a set of MODIS images acquired in August-October 2008 and successfully estimated both an area and a perimeter of the floe of interest over the given time period.

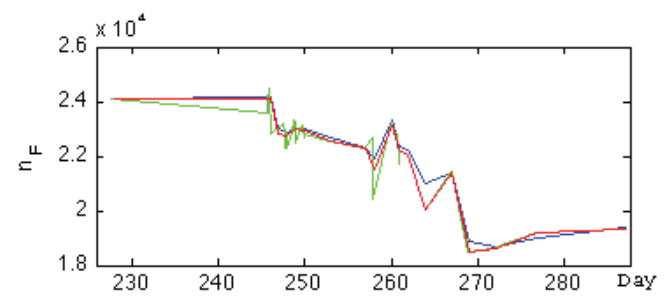

(a)

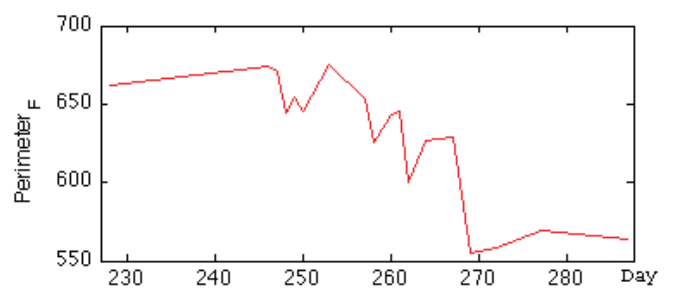

(b)

Fig. 4. (a) Ice floe area (number of pixels) as a function of time: Before post-filtering averaged for every day (blue); After post-filtering (green); After post-filtering averaged for every day (red). (b) Ice floe perimeter as a function of time, averaged for every day.

\section{REFERENCES}

[1] H. Le Treut, C. Mauritzen, A. Mokssit, T. Peterson, M. Prather, R. Somerville, U. Cubasch, and Y. Ding, Historical Overview of Climate Change, pp. 95-122, Cambridge University Press, 2007.

[2] HongJiang Zhang, "Automatic tracking ice floe from satellite imagery abstract via invariant moment matching," in Proc. of IGARSS'92, Houston, TX, May 1992, pp. 582-584.

[3] D. Haverkamp and C. Tsatsoulis, "Using temporal information in an automated classification of summer, marginal ice zone imagery," in Proc. of IGARSS'96, Lincoln, Nebraska USA, May 1996, pp. 109-111.

[4] P. Yu, "Segmentation of RADARSAT-2 dualpolarization sea ice imagery," Master thesis, University of Waterloo, Ontario, Canada, 2009.

[5] J.-M. Beaulieu and M. Goldberg, "Hierarchy in picture segmentation: a stepwise optimization approach," IEEE Trans. Pattern Analysis and Machine Intelligence, vol. 11, no. 2, pp. 150-163, Feb 1989.

[6] R. Wolfe and M. Nishihama, "Trends in modis geolocation error analysis," in Proc. of SPIE - Earth Observing Syst. XIV, 2009, vol. 7452, p. 745 20L.

[7] P. Soille, Morphological Image Analysis, SpringerVerlag, Heidelberg, 2nd edition, 2003. 